\title{
Adaptaciones informales y nociones de lo público frente a la densificación urbana*
}

Fecha de recepción: 20 de noviembre de 2017 | Fecha de aprobación: 7 de noviembre de 2018 | Fecha de publicación: 30 de julio de 2019 Cristina Isabel Dreifuss-Serrano

\begin{tabular}{l|l|l|}
\hline Universidad de Lima, Perú & ORCID: 0000-0001-6540-3167 & cdreifus@ulima.edu.pe
\end{tabular}

Ángeles Margarita Maqueira-Yamasaki

Universidad de Lima, Perú

ORCID: 0000-0003-1111-1075

Resumen El estudio se centra en el impacto del crecimiento inmobiliario reciente en Lima, en el uso que se le da al espacio urbano, y cómo éste afecta las dinámicas sociales, la cohesión de vecindarios y la identidad de las personas en relación a su lugar de residencia. A partir del estudio del desarrollo de un barrio en proceso de densificación inmobiliaria se identifican patrones de uso del espacio urbano y su evolución con los cambios del vecindario, así como la llegada de una nueva población, más numerosa. Muchas de las premisas de base, en cuanto al déficit de espacio público y a los cambios de uso de la zona, son evidentes desde un punto de vista cuantitativo; sin embargo, las encuestas demuestran que la percepción de la población local no siempre coincide con esto. Así mismo, se establece un diálogo entre realidad y percepción, el cual permite delinear cuáles eventos permanecen y cuáles están siendo reemplazados.

Palabras clave crecimiento urbano, densificación, espacio público, informalidad, infraestructura, vivienda 


\section{Informal Adaptations and Notions}

of the Public regarding the Urban Densification

Abstract This study focuses on the impact of the real estate growth in Lima, how the urban space is being used and how all this affects the social dynamics, the neighborhood cohesion and the individual's identity in relation to his/her place of living. By studying the development of a neighborhood undergoing a real estate densification process, this work identifies the patterns of public space use and its evolution amidst the neighborhood changes. It also studies the arrival of a new and bigger population. Many of the basic premises concerning the public space deficits and the changes in the land use therein become evident under a quantitative viewpoint. However, the surveys show that the perceptions among the local population do not always match those facts. Likewise, a dialogue has been started between reality and perception, which allows define the events that remain and those being replaced.

Keywords urban growth, densification, public space, informality, infrastructure, housing

\section{Adaptações informais e noções}

do público frente à densificação urbana

Resumo O estudo foca-se no impacto do acréscimo imobiliário recente de Lima, no uso dado ao espaço urbano, e como isso afeta as dinâmicas sociais, a coesão de vizinhanças e a identidade das pessoas em relação a seu local de residência. A partir do estudo do desenvolvimento de um bairro em processo de densificação imobiliária identificam-se padróes de uso do espaço urbano e sua evolução com as mudanças da vizinhança, bem como a chegada de nova população, mais numerosa. Muitas das premissas básicas, relativas ao déficit de espaço público e mudanças de uso da área, são evidentes desde o ponto de vista quantitativo; no entanto, pesquisas mostram que a percepçáo da populaçáo local nem sempre coincide com isso. Da mesma forma, estabelece-se um diálogo entre realidade e percepção que permite delinear quais eventos permanecem e quais estão sendo substituídos.

Palavras chave acréscimo urbano, densificação, espaço público, informalidade, infraestrutura, habitação 


\section{Introducción}

En contextos de desarrollo urbano acelerado, impulsado por iniciativas privadas, el espacio público rara vez es considerado durante la transformación de viviendas unifamiliares en edificios multifamiliares. Este proceso de densificación no está acompañado por un desarrollo equilibrado de políticas para el uso, mejora o adaptación de áreas públicas aledañas. Sin embargo, los habitantes continuarán utilizando el espacio público existente con acciones que se convierten en estrategias de apropiación del espacio, mientras que, al mismo tiempo, buscarán satisfacer necesidades específicas, individuales o colectivas.

Las actividades dependen de las posibilidades físicas que el espacio mismo ofrece. Son frecuentes los procesos informales que adaptan las condiciones dadas en una plaza o camino existente para adecuarse a los requerimientos o preferencias de la gente.

El estudio ha seleccionado un eje de dos kilómetros de longitud en un área céntrica de Lima (avenidas Gregorio Escobedo, y Pardo y Aliaga, en los distritos de Jesús María y Magdalena). En el eje se da un proceso de densificación, en el cual grandes viviendas unifamiliares han sido demolidas; y los terrenos, utilizados para construir edificios de vivienda de veinte pisos, que ofrecen una oferta más económica de apartamentos pequeños. Esto no sólo produce un aumento dramático en la población del distrito, sino también un cambio en la composición familiar y las costumbres sociales. Como se ve en el mapa de la figura 1, las primeras manzanas del eje ya han sido casi completamente reemplazadas por edificios, mientras que el extremo opuesto aún alberga viviendas unifamiliares tradicionales. Es por esto que el estudio analiza ambas realidades en una suerte de línea de tiempo, dada por el desarrollo progresivo en el área.

El objetivo de la investigación es analizar el comportamiento en el espacio público, las variaciones en la composición familiar y en la ocupación del territorio, y la percepción de los usuarios con respecto a los cambios recientes. 


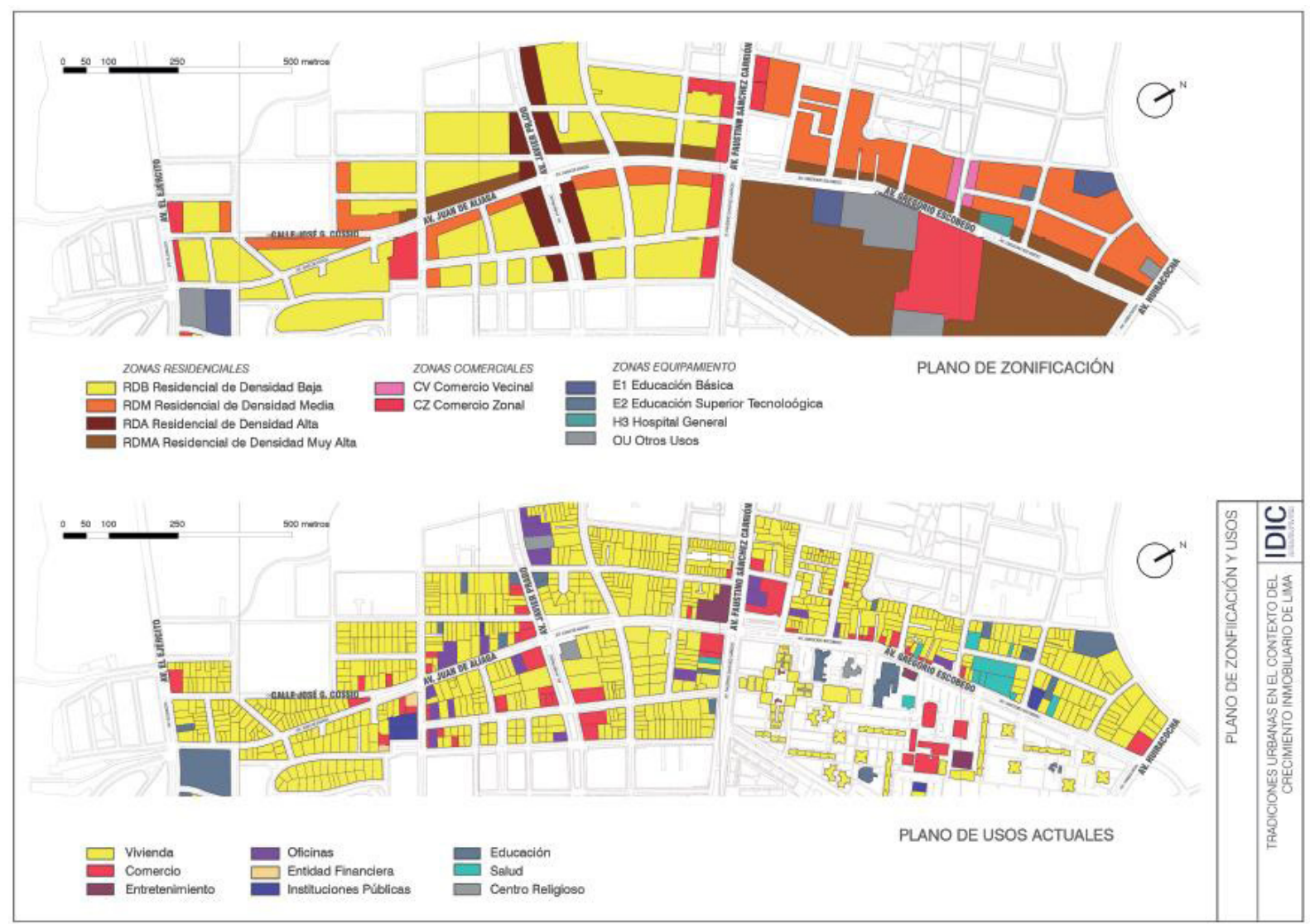

Figura 1. Zona de estudio. Plano de usos de suelo actuales, por lote Fuente: elaboración propia

\section{Antecedentes}

Según la encuesta anual del Observatorio Lima Cómo Vamos, en 2010, el 56,9\% de los limeños creía que los nuevos edificios y viviendas hacían que la ciudad se viera "más bonita". Para el año 2014, este porcentaje ha descendido a un $43 \%$. Sólo el 12\% considera que la falta de árboles y áreas verdes es un problema importante, y únicamente el 20\% afirma que sus hijos salen a jugar a la calle. En general, el 54,6\% de los limeños considera que se tiene un mal cuidado del espacio público, a pesar de que un 70,3\% manifiesta tener como principal actividad recreativa ir a pasear a parques (Lima cómo vamos, 2013).

A raíz del crecimiento inmobiliario, muchos espacios urbanos han cambiado. La densidad ha generado nuevas formas de habitar en unidades de vivienda más pequeńas y en mayor cantidad, que proponen al usuario nuevos modos de recorrer y vivir la ciudad. Lamentablemente, dentro de este desarrollo acelerado, el desarrollo de lo privado no corresponde con el del espacio público.

Esta situación plantea el reto de un crecimiento acorde de dicho espacio público. Los entes regularizadores de la ciudad podrían plantear un aporte desde el ámbito de la inversión inmobiliaria privada para mejorar el equipamiento del espacio urbano, pero ¿cuáles son aquellos aspectos de la vida urbana en los que este equipamiento debe enfocarse? Al asumir que el espacio público es necesario para el hombre (Arendt, 2011/1958; Jacobs, 2011/1961), y que una de sus funciones principales es la de albergar actividades individuales 
y colectivas de trascendencia, se debe entender cómo se utiliza y qué tipo de actividades permite o propicia.

Los estudios sobre el espacio público que se centran en lo construido plantean lineamientos para su diseño, posibles lecturas de apreciación y análisis de situaciones actuales. Son relevantes los trabajos analíticos de Kevin Lynch (2001/1984), Colin Rowe y Fred Koetter (1998/1981) y Edward Soja (2000), así como los más descriptivos, que incluyen estudios de casos, como la obra de Gordon Cullen (1974), Rob Krier (1981), Antoine Bailly (1979), Jan Gehl (2006/2004) y Angelique Trachana (2008), quienes se han preocupado por estudiar, clasificar y definir aquellos elementos físicos que conforman la ciudad.

Estudios recientes han profundizado sobre los usos espontáneos del espacio público frente a una inadecuada o insuficiente planificación (Carrión, 2007; Cuervo-Calle y Herrán-Cuartas, 2013; Franck y Stevens, 2007; Ludeńa-Urquizo, 2004; Páramo y Burbano-Arroyo, 2014; Tokeshi y Takano, 2007), de la mano del crecimiento acelerado y la densificación de la urbe (Beer, Delshammar y Schildwatch, 2003; Borja y Muxí, 2000; Páramo et al., 2018; Pérez-Bourzac, 2018; Vega-Centeno, 2017).

Estudios influyentes sobre el espacio público han sido muchas veces elaborados por profesionales de otras disciplinas. Desde el periodismo, Jane Jacobs (2011/1961) realiza un estudio de las dinámicas urbanas en Manhattan, en su obra The Death and Life of Great American Cities, mientras que la sociología y la filosofía plantean, por su parte, claves de lectura de la ciudad desde el punto de vista del ciudadano, su identificación con el entorno y la construcción de identidades individuales y colectivas (Arendt, 2011/1958; Lefebvre, 1973/1970).

El fenómeno urbano no sólo está compuesto de elementos físicos, la variable humana es un fuerte factor determinante en la manera como se producen y utilizan las ciudades. Las costumbres, la cultura, los imaginarios (Huffschmid, 2012), la identidad y la cosmovisión que conforman el ámbito de lo intangible, son variables que han de considerarse de forma paralela a las variables físicas y morfológicas (Agudelo-Castañeda, 2011).

La ciudad es un fenómeno autoorganizado (Brillenbourg, Feireiss y Klumpner, 2005; Urban-Think Tank, 2013), una red compleja de decisiones interconectadas. Se da una relación causa y efecto en dos direcciones: la ciudad influye en el habitante y el habitante en la ciudad, y el pensamiento y las acciones dejan una huella legible en los edificios de la ciudad (Johnson, 2001). Los reglamentos y planes urbanos que han triunfado, lo han hecho porque han entendido la interacción entre los individuos que caracterizan a cada colectivo urbano y el impacto de dicha interacción en el espacio público, y viceversa.

El "boom inmobiliario" de inicios del siglo XXI en Lima, ha despertado la curiosidad, no sólo de arquitectos, sino también de inversionistas, economistas y políticos. "Estamos atravesando por uno de esos períodos a los que la historia se referirá como el momento en el que se creó un nuevo tipo de ciudad, cuando la capital adquirió un nuevo rostro" (Roca-Mora, 2013, p. 68). Lamentablemente se trata de una ciudad poco planificada, en la que la realidad, normalmente, precede a la normativa, y la consecuencia es una dramática reducción en la cantidad de espacios vacíos en la ciudad.

Por un lado, en distritos céntricos y tradicionales, como Miraflores, San Isidro, Magdalena y Jesús María se empezarían a comprar casonas para poder levantar edificios. “(...) de cada cinco proyectos que se desarrollan en estos distritos, cuatro son ejecutados en terrenos donde hubo una casona. Los terrenos tienen un rango de $400 \mathrm{a}$ 600 metros cuadrados" (López, 2011, p. 30). Por otro, en zonas periféricas, las antiguas áreas agrícolas se volcaron a satisfacer la necesidad de la 
vivienda (López, 2011). Sólo considerar el crecimiento de las alturas de las edificaciones aledańas a las vías resulta en un cambio dramático en la escala y en la experiencia cotidiana de la ciudad, donde las "iniciativas inmobiliarias se apoyan en la aún persistente sensación de miedo generalizado que en parte produjo el terrorismo en Lima, para legitimar una cierta aversión a la calle como espacio para la vida social" (Vega-Centeno, 2017, pp. 30-31).

Esta densificación -en términos de habitación y aumento en alturas, y porcentajes de ocupación de los lotes- no ha tenido un equivalente ni cualitativo (aumento de áreas) ni cuantitativo (mejora de servicios ofrecidos o infraestructura), en cuanto a las áreas públicas en los vecindarios afectados. Aumenta la vivienda, la cantidad de habitantes y la presencia de lo construido, pero los parques, los espacios libres entre las viviendas y las vías siguen manteniendo la configuración diseñada para urbanizaciones de vivienda unifamiliar (ver figuras 2, 3, 4 y 5).

Sin embargo, los análisis y las conclusiones respecto del crecimiento inmobiliario en Lima reflejan una actitud positiva ante la continuidad del cambio. Afirmaciones como "existe todavía mucho margen para continuar la expansión" (López, 2011, p. 32) y "aún hay espacio para crecer" (Roca-Mora, 2013, p. 68) evidencian una continua inversión y posibilidad de adquisición de vivienda propia como un indicador de progreso para muchos limeños. No obstante, nos estamos olvidando del espacio urbano.

Se percibe, además, que los nuevos proyectos de vivienda ofrecen áreas sustancialmente menores que las unidades a las que reemplazan, por lo que el espacio vital de las familias se ve dramáticamente reducido. Una variable importante que concierne al ámbito del espacio público tiene que ver con la noción de área común en la compraventa de un bien inmueble. "(...) los edificios hoy tienden a ofrecer departamentos más pequeños, pero con espacios compartidos más grandes. La incorporación de gimnasios y áreas verdes es cada vez mayor en grandes proyectos, dándole un plus al valor del inmueble" (Roca-Mora, 2013, p. 70).

El espacio público, exterior, al aire libre es insuficiente y de baja calidad (Vega-Centeno, 2017), por lo cual, las empresas que negocian con el habitar necesitan ofrecer un valor agregado que satisfaga la necesidad de espacio libre, compartido y social, de sus posibles compradores. Dado que la ciudad suele ser percibida como peligrosa (Lima cómo vamos, 2013), y es común que el espacio público sea considerado como un lugar de riesgo, las familias prefieren espacios semipúblicos -compartidos por los vecinos de un mismo edificiodonde sienten una mayor seguridad. 


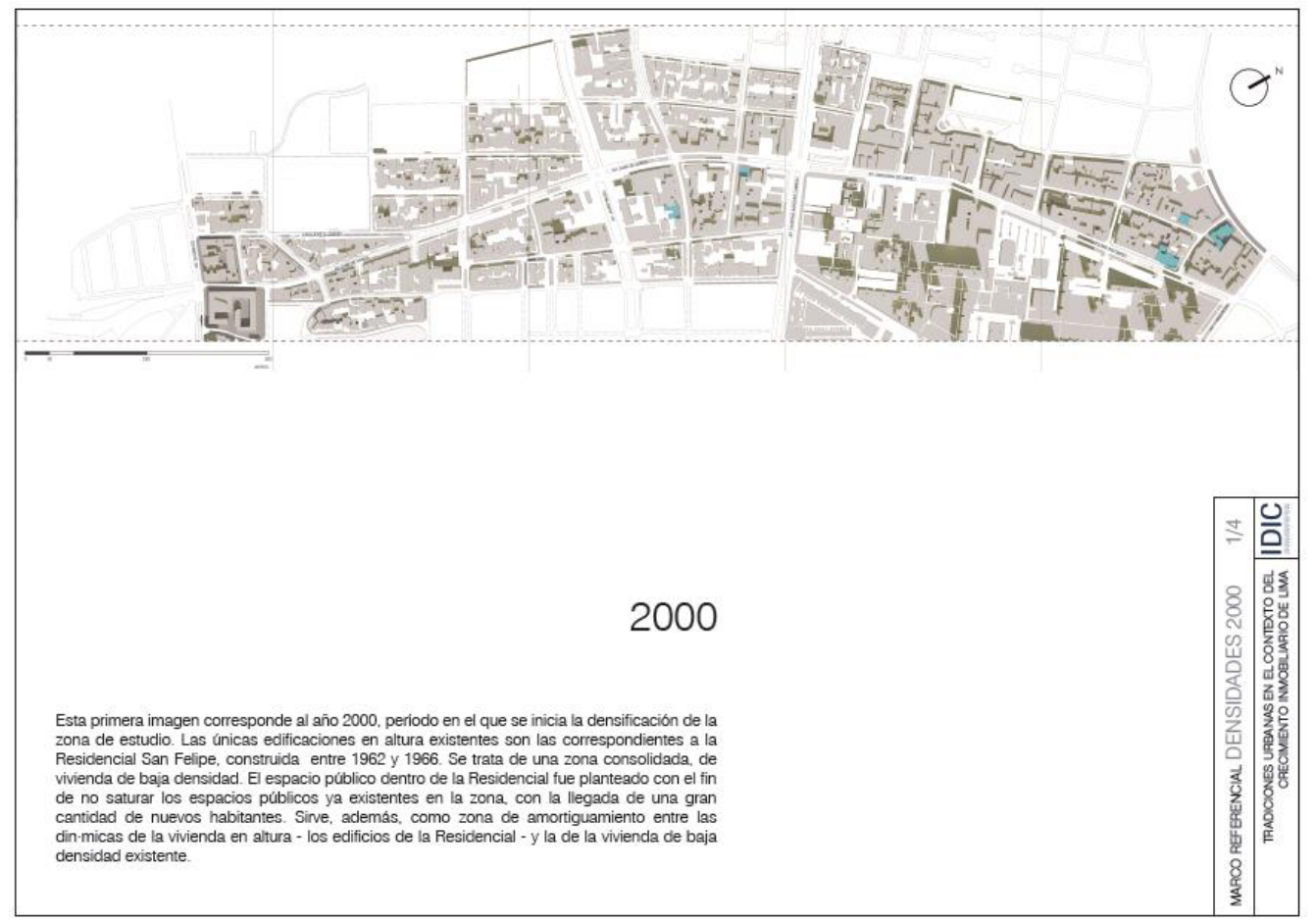

Figura 2. La zona de estudio en 2000 , inicio del crecimiento inmobiliario acelerado Fuente: elaboración propia en base a aerofotografías del Instituto Geográfico Nacional

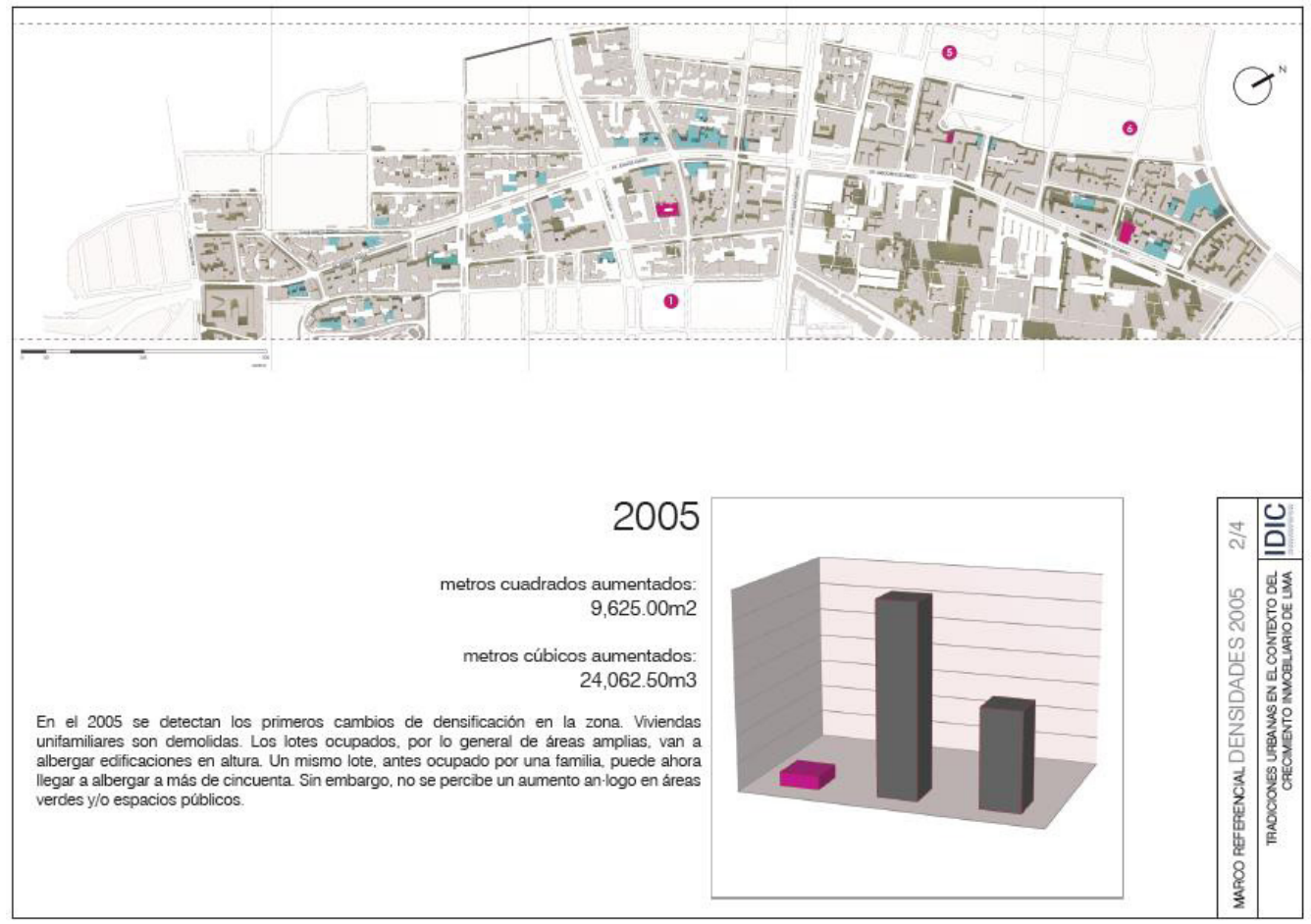

Figura 3. La zona de estudio en 2005

Fuente: elaboración propia en base a aerofotografías del Instituto Geográfico Nacional 


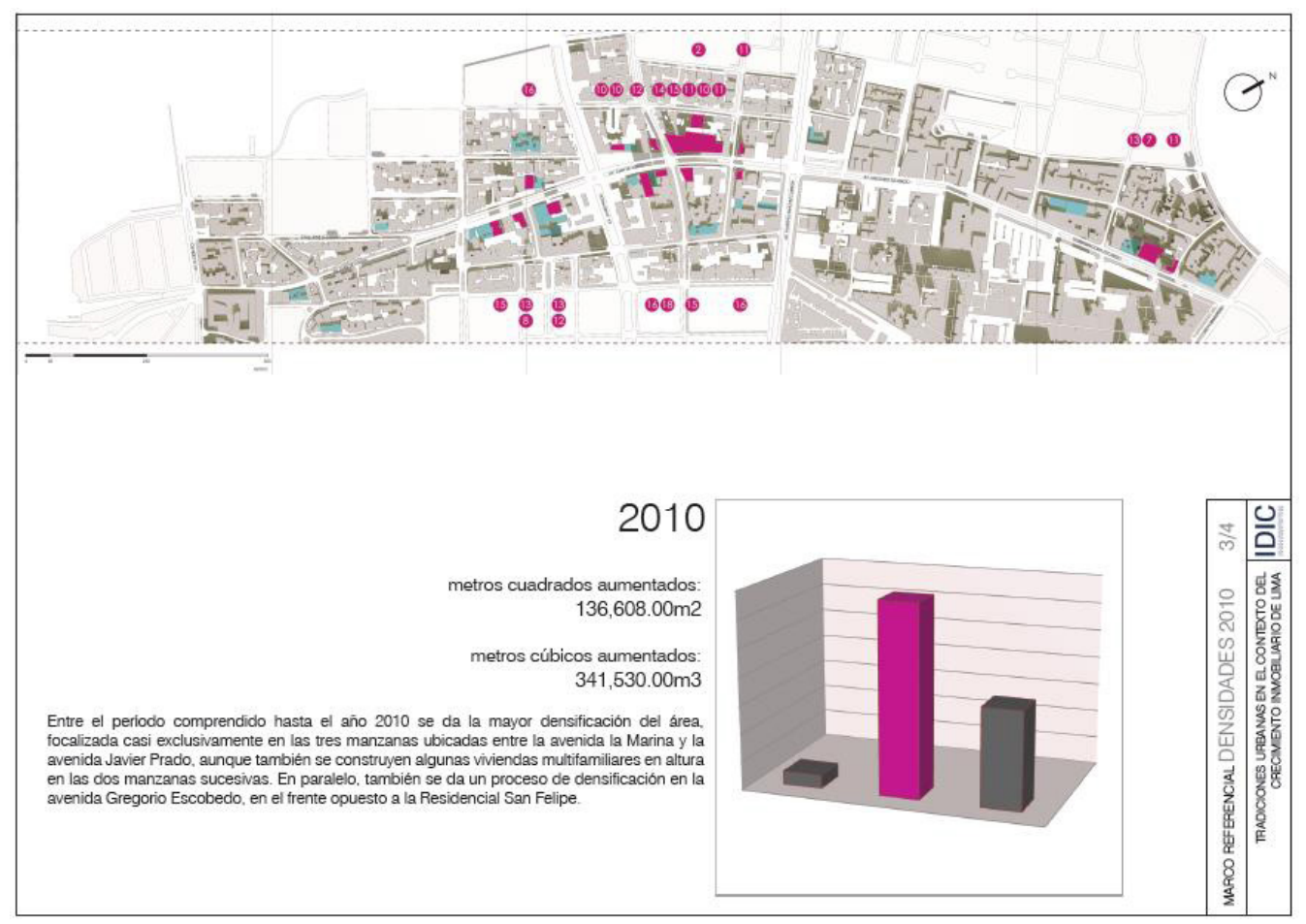

Figura 4. La zona de estudio en 2010

Fuente: elaboración propia en base a aerofotografías del Instituto Geográfico Nacional

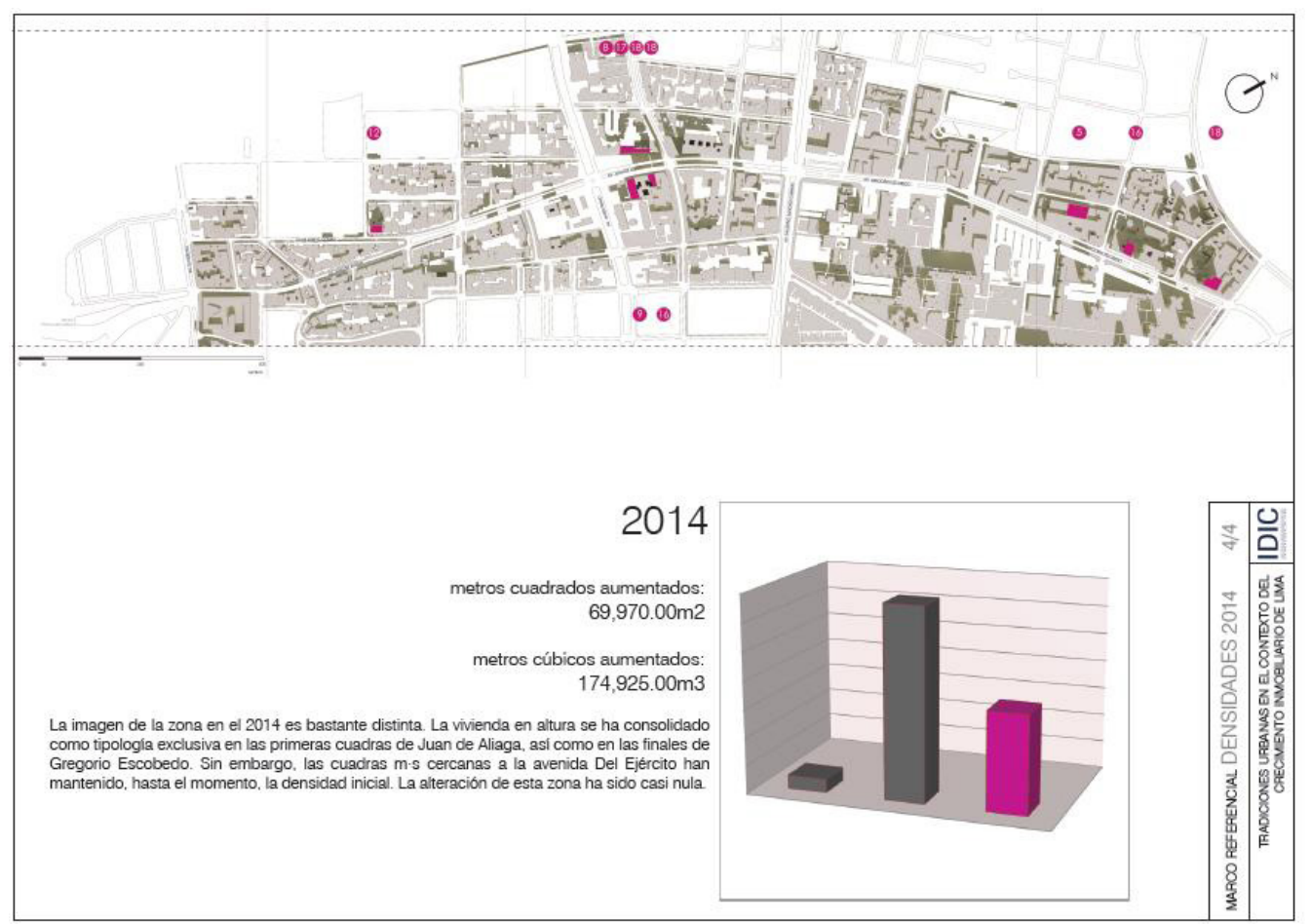

Figura 5. La zona de estudio en 2014

Fuente: elaboración propia con base en aerofotografías del Instituto Geográfico Nacional 


\section{Metodología}

El estudio se articula con base al mapeo de los eventos sociales más relevantes de la zona, los cuales están ligados al uso recurrente de determinados espacios urbanos, y a entrevistas y encuestas realizadas a usuarios de la zona elegida. De la información de ambas fuentes se identifican patrones recurrentes, nuevos eventos, eventos consolidados y eventos desaparecidos. Así, se establecen relaciones entre los espacios arquitectónicos como medio físico y los eventos que se desarrollan en ellos.

El eje de las Avenidas Gregorio Escobedo y Juan de Aliaga fue dividido en tres zonas. Cada una de estas corresponde a un nivel distinto de consolidación de la trama urbana, usos de suelo y tipos de vivienda. La zona uno corresponde al tramo de la Av. Gregorio Escobedo, entre el Jr. Huiracocha y la Av. F. Sánchez Carrión. Observaciones preliminares indicaron que en esta zona se pueden encontrar dos tipos de usuarios del espacio público diferenciables: los habitantes y usuarios del espacio de la Residencial San Felipe, quienes suelen vivir en esta o en cercanías, y los clientes de los negocios y oficinas ubicados al otro lado de la calle. La zona dos empieza en el cruce de la Av. Juan de Aliaga con la Av. F. Sánchez Carrión, hasta el cruce con la Av. Javier Prado. Esta es la zona donde el desarrollo inmobiliario ha sido mayor. Las viviendas unifamiliares de dos pisos han sido remplazadas casi en su totalidad por edificios multifamiliares de más de 15 pisos. En contraste, se encuentra la menor cantidad de espacio público. La zona tres abarca desde el cruce de la Av. Juan de Aliaga con la Av. Javier Prado, hasta el cruce de la primera con la Av. Del Ejército. Las viviendas son en su mayoría unifamiliares, y la presencia de actores externos es considerablemente menor, por tratarse de una zona con un uso de suelo casi exclusivamente residencial.
El objetivo principal de la encuesta es identificar comportamientos a patrir de preguntas relacionadas con las actividades de los usuarios -residentes o no- en el espacio público. La noción de espacio público considerada incluye, no sólo parques y plazas, sino también calles, veredas, bermas y espacios libres entre los edificios (Vega-Centeno, 2017). Con el fin de alcanzar este objetivo, se elaboró una tabla de variedad que incluye todos los posibles espacios públicos de la zona, para la cual se tuvo en cuenta un amplio radio de acción de 800 metros a la redonda del eje estudiado.

El primer método de aproximación a los usuarios de la zona fue la entrevista no parametrada con usuarios permanentes y temporales de la zona, la cual permitió establecer un marco de referencia para la elaboración de una encuesta más exhaustiva. Las personas entrevistadas realizan actividades que fuerzan una permanencia en la zona durante largos períodos de tiempo: empleados, dueños de locales comerciales, vecinos, personal de seguridad, personal de mantenimiento público, entre otros.

La encuesta permite corroborar, de modo cuantitativo, la existencia de los patrones mencionados, así como su frecuencia y su posible variación en el tiempo. Se mantuvo la división del área de estudio en las tres zonas previamente identificadas y, considerando la población del área, se tomó una muestra de 100 personas por zona, a las que se encuestó por intercepción en la vía pública.

Con el objetivo de "evaluar la manera cómo las características físicas de las ciudades son experimentadas y entendidas por personas ordinarias" (Groat y Wang, 2002, p. 227), los resultados de la encuesta son comparados con las observaciones realizadas en campo, levantadas con base en fotografías periódicas en distintos espacios públicos identificados en la zona. 


\section{Resultados}

Se identificaron los lugares utilizados como espacios públicos, tanto lo planificados - parques, centros deportivos-, como aquellos que son utilizados a pesar de tener un diseño que no contempla los usos observados -bermas, esquinas.

Un porcentaje importante de los entrevistados $(40 \%)$ recorre la zona porque vive en ella. Esta presencia relativa de visitantes de otras zonas, en un principio, sería un factor positivo para el fortalecimiento de la identidad barrial y el establecimiento de nexos entre los vecinos. Sin embargo, es también alto el porcentaje de personas (34\%) que se encuentran en la zona por estudio o trabajo, sobre todo en las zonas 2 y 3 . Esto trae al barrio un movimiento constante, que demanda mejores condiciones en el espacio público; sin embargo, esta población flotante es percibida por los vecinos como un elemento de riesgo (ver figura 6).

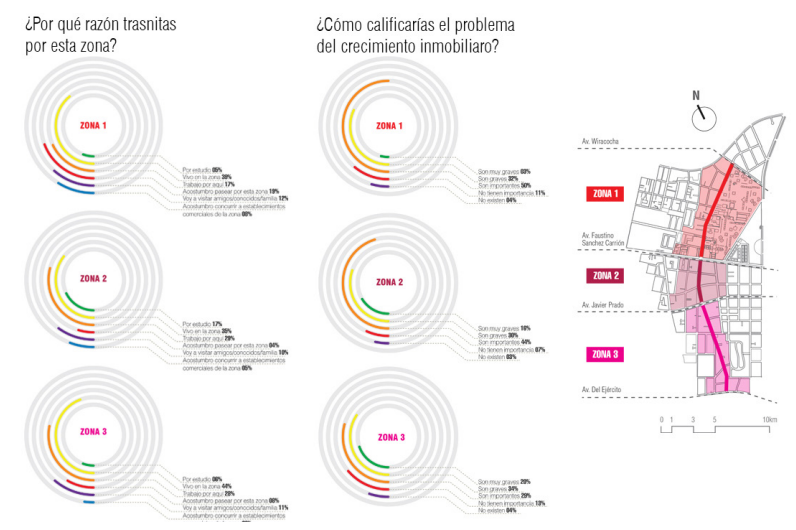

Figura 6. Encuestas, variación de las respuestas según zonas dentro del área de estudio

Fuente: elaboración propia

La frecuencia de uso de parques es bastante alta: $72 \%$ de los encuestados va una vez por semana, o más, a un parque, y el $24 \%$ lo hace a diario. El uso de plazas plantea un panorama dramáticamente opuesto: más de la mitad de los encuestados afirma no utilizarlas. Por un lado, esto puede deberse a que en la zona no hay plazas, a excepción del ágora de la Residencial San Felipe; por otro, las plazas no permiten muchas de las actividades que se realizan en los parques, como el paseo de perros o el juego de niños.

En la zona de estudio, las bermas centrales son un caso interesante. A diferencia de lo ocurrido en avenidas como la José Pardo, en Miraflores o Salaverry -tangencial a la zona de estudio y donde espacio cuenta con un tratamiento de bancas, farolas y ciclovías- la berma central de la avenida Juan de Aliaga sólo tiene jardín y algunos árboles. Si bien un porcentaje alto de personas dice nunca utilizar la berma (41\%), existe una cantidad considerable (46\%) que sí la utiliza, al menos una vez a la semana, mientras que un cuarto de la población la utiliza a diario. Esto, posiblemente, se debe a lo conveniente para pasear mascotas sin tener que alejarse mucho de la vivienda. A pesar de la falta de equipamiento y condiciones adecuadas de accesibilidad, la berma central es un espacio público con mucho potencial.

El nuevo centro comercial Real Plaza Salaverry es usado por un gran porcentaje de vecinos como lugar de paseo con una cierta frecuencia, 30\% señala ir una vez por semana. Los centros comerciales en la ciudad contemporánea "suplantan la función histórica de la plaza y la calle tradicional para transformarse en lugares que representan la utopía y aspiraciones de las clases medias y altas" (Pérez-Bourzac, 2018, p. 135). Una de las razones por las que los centros comerciales son considerados como lugares de paseo es la percepción de mayor seguridad con respecto al espacio público. A nivel de Lima Metropolitana, la encuesta anual "Lima cómo vamos" ha identificado que la delincuencia y la inseguridad ciudadana son percibidos como el problema más importante que afecta la calidad de vida (Lima cómo vamos, 2013, 2018). En concordancia con este hecho, frente a la afirmación "prefiero pasear en un centro comercial que en las calles", 57\% de 
los encuestados se manifiesta de acuerdo; y el $43 \%$, en desacuerdo.

A pesar de lo expresado en algunas entrevistas, $40 \%$ de los encuestados expresa que el vecindario es tranquilo y seguro. Pocos (4\%) se refieren directamente a la relación con los vecinos, por lo que la percepción de tranquilidad está más relacionada con el espacio que con las relaciones interpersonales. Sólo un 14\% señala los parques y las áreas verdes como un aspecto positivo de la zona.

Dentro de los aspectos negativos, el principal $(60,7 \%)$ es la inseguridad, percibida de igual manera por personas de diferentes edades. Es interesante comparar este dato con la encuesta metropolitana del año en cuestión, en la que el factor inseguridad es señalado como uno de los principales problemas de la ciudad por el 83,5\% de la población (Lima cómo vamos, 2013). Comparativamente, la zona de análisis es percibida por sus usuarios como menos insegura. La contaminación ambiental y el tráfico son factores importantes, pero de menor relevancia. Este último es especialmente notado por personas mayores (a partir de los 38 años). Contrario a lo que plantean las teorías estudiadas, la falta de equipamiento juegos para niños, ciclovías, bancas- no se considera como un aspecto especialmente negativo de la zona $(0,7 \%)$. Este factor sólo fue mencionado por los encuestados entre 18 y 27 años.

Si bien el crecimiento inmobiliario es considerado el principal problema de la zona, $75 \%$ de los encuestados considera que el vecindario ha mejorado en los últimos años, mientras que un 18\% opina lo contrario. Este resultado es más dramático en la zona 2 , donde $84 \%$ está de acuerdo con esta afirmación; sin embargo, esta también es la zona con mayor población nueva, por lo que es posible que la respuesta haya sido dada por personas que no recorrían o habitaban la zona hace 10 ańos.
A pesar de la percepción de seguridad y confianza, manifiesta en preguntas anteriores, cuando se pide a los encuestados que describan qué entienden por espacio público, $53,2 \%$ mencionan inseguridad, seguido de un $15,5 \%$ que se refiere a contaminación ambientallsonora, y de un $13,8 \%$ que menciona exceso de tráfico alrededor. El concepto de espacio público tiene claras connotaciones negativas, que son comunes a todas las edades y zonas (ver figura 7).

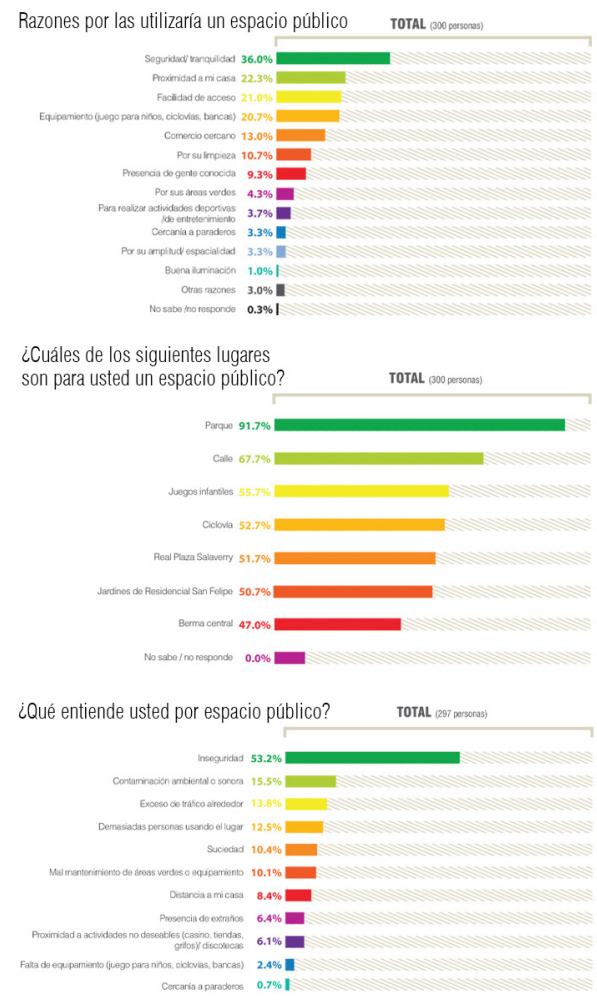

Figura 7. Encuestas, preguntas relacionadas a la percepción del espacio público

Fuente: elaboración propia

$\mathrm{Al}$ referirse a los problemas, los vecinos de la zona no consideran que haya una ausencia de áreas verdes; de hecho, el $81 \%$ considera que esto no es un problema. Posiblemente esto se deba a que la suma de bermas, pequeños jardines de retiro, parques y las zonas comunes de la Residencial San Felipe suman una cantidad considerable de 
área verde, que contrasta con la mayoría de distritos de Lima. Esta pregunta, sin embargo, no indaga sobre el uso de dicha área, sólo sobre su percepción, la cual puede limitarse a lo visual.

En cuanto a la relación con los vecinos, los resultados son altamente contrastantes. El 55\% manifiesta conocer a buena parte de sus vecinos, mientras que el $43 \%$ está en desacuerdo con esta afirmación. La polarización de esta respuesta se da casi por igual en las zonas 2 y 3 . Sin embargo, la zona 1 se distingue con un $65 \%$ que dice conocer a sus vecinos, versus un 33\% que no. Esta particular puede deberse al tipo de viviendas en la Residencial San Felipe, en donde se da una mayor participación de los vecinos en la toma de decisiones.

Las razones para la utilización de un determinado espacio público son varias. La principal (36\%) es seguridad/tranquilidad, seguida por proximidad a la vivienda (22,3\%), facilidad de acceso (21\%) y equipamiento -juegos para niños, ciclovías, bancas (20,7\%). Otros aspectos importantes son existencia de comercio cercano, limpieza y presencia de gente conocida. Este último factor es de especial relevancia para la población joven, entre 15 y 17 años (20\%).

En oposición, las razones para no usar el espacio público son inseguridad $(52,7 \%)$, contaminación ambiental o sonora $(15,3 \%)$, exceso de tráfico alrededor $(13,7 \%)$ y demasiadas personas usando el lugar (12,3\%). También se menciona suciedad, mal mantenimiento de áreas verdes o equipamiento urbano, distancia al espacio público y presencia de extrańos. En todos los grupos de edades la inseguridad es el factor más relevante.

\section{Conclusiones}

Si bien la subdivisión del área en zonas y la clasificación de los fenómenos urbanos observados en distintos tipos constituyen herramientas de análisis necesarias, dada la complejidad y la situación cambiante del área de estudio, es importante señalar que la ciudad debe entenderse como un sistema en el que la articulación de espacios es fundamental.

Una de las principales características identificadas es la mencionada diferenciación entre sus partes. La existencia de barreras en el cruce difícil de las avenidas y el gran contraste entre los usos de suelo hace que este eje, que geográficamente parece unificado y unificador, sea en la práctica un conjunto de barrios poco articulados entre sí.

Observamos en el uso del espacio público una combinación de prácticas formales e informales. El caso que más resalta es el de la berma central, que es usada a diario y de manera breve para pasear los perros. Gente que trabaja en la zona también hace uso de ella a la hora de almuerzo, como área de descanso (ver figura 8). Esta ocupación informal toma los recursos a disposición para desarrollar las actividades de la mejor manera posible, las tomas de sumideros públicos de agua se convierten en bancas; las sombras de los árboles, en lugares para estar; los jardines, en pequeńos parques o espacios para hacer ejercicio. Las actitudes corresponden a una suerte de domesticación del espacio público (Cuervo-Calle y Herrán-Cuartas, 2013). El número de actividades, tanto formales como informales, y su variedad no permite establecer patrones característicos para las zonas 1 y 2 . Es en la zona 3 donde se encuentra un uso casi inexistente del poco espacio público a disposición (ver figura 9). 

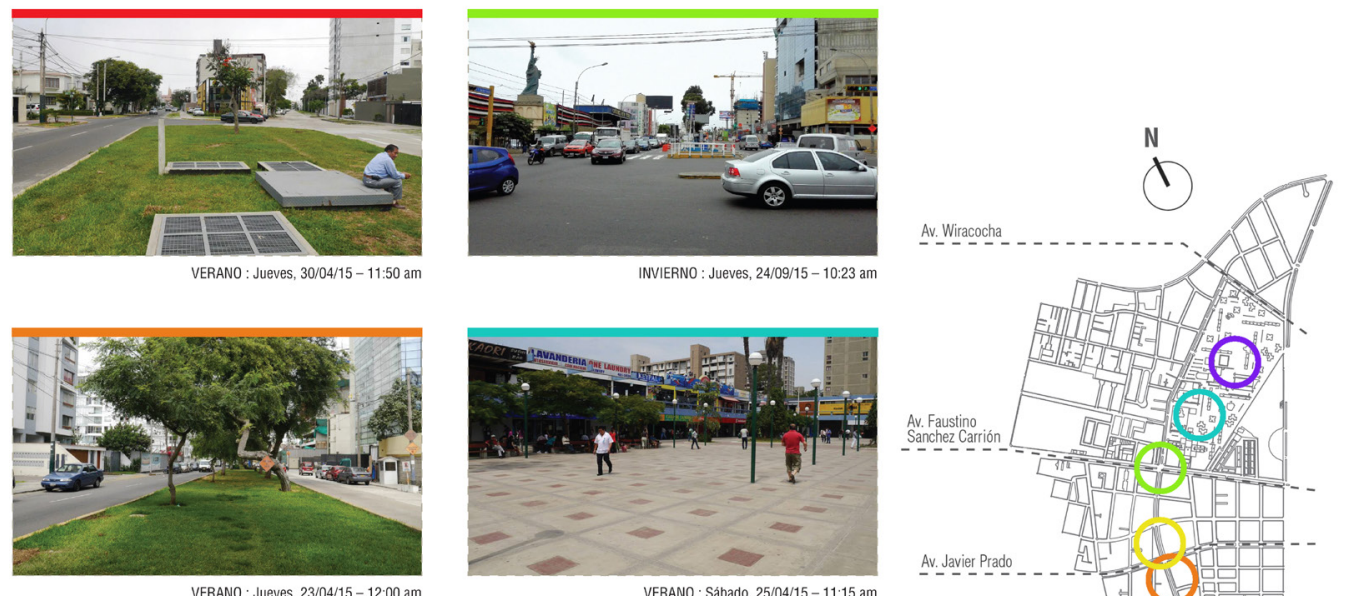

VERANO : Jueves, 23/04/15-12:00 an
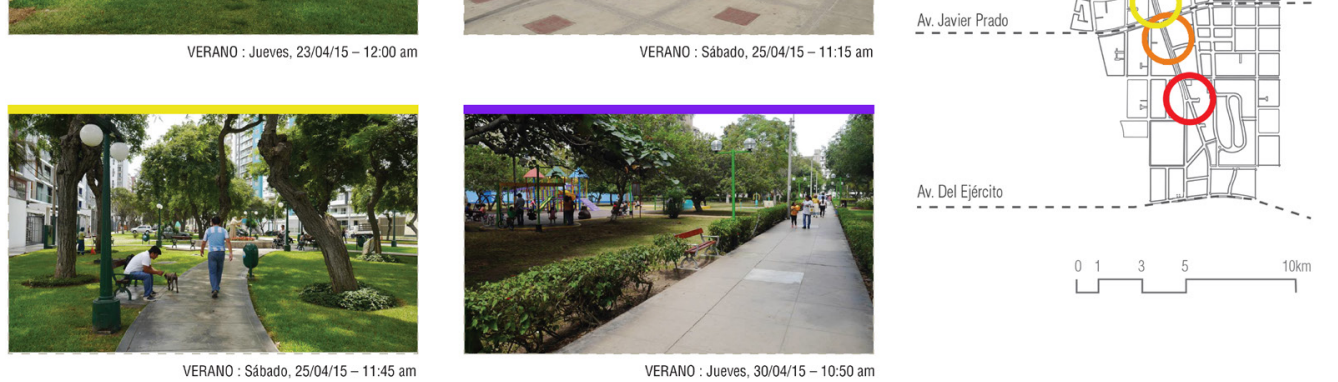

Figura 8. Fotografías de las zonas de estudio

Fuente: elaboración propia

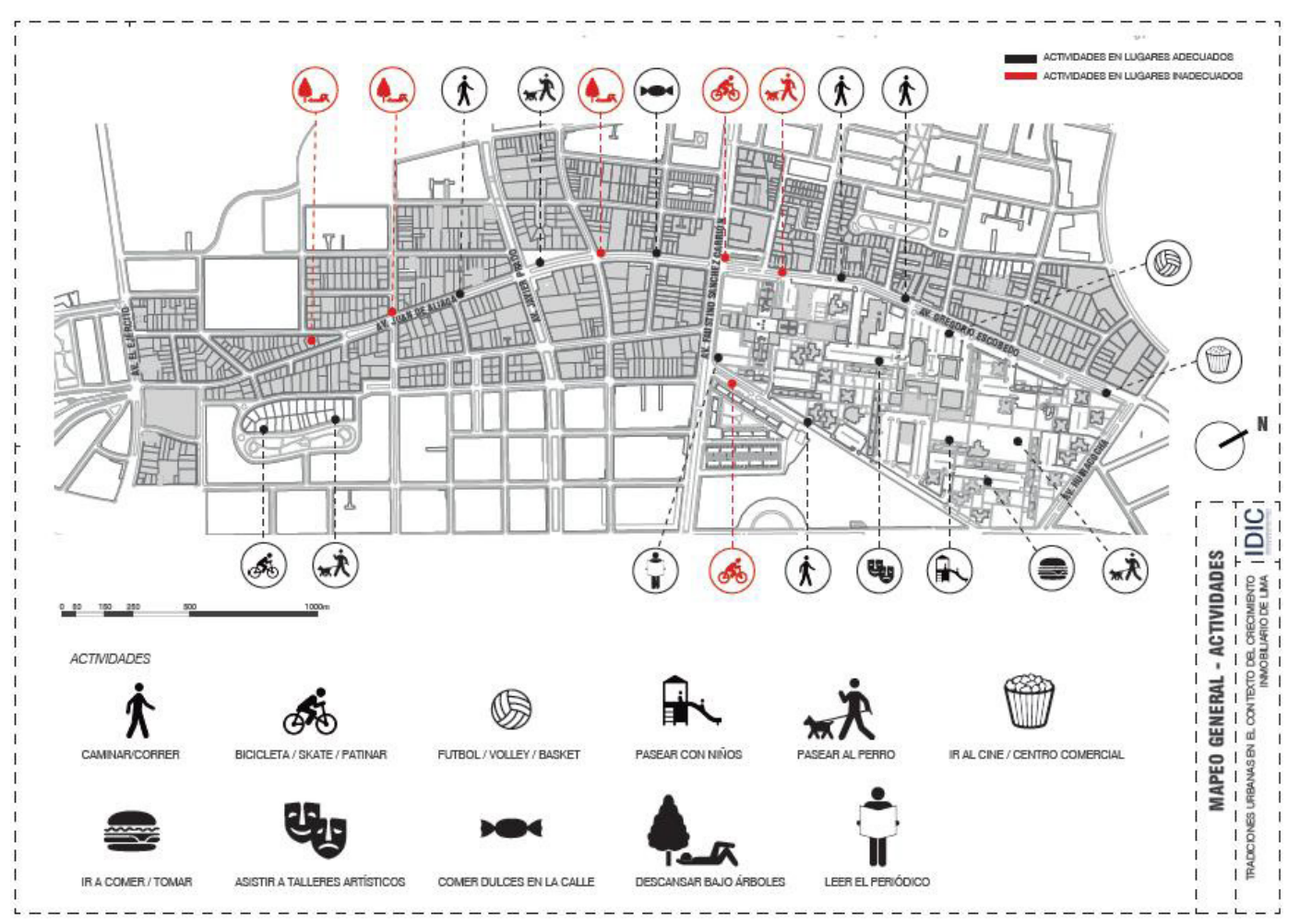

Figura 9. Mapeo general - actividades realizadas en el espacio público del área de estudio Fuente: elaboración propia 
Las encuestas han seńalado que los vecinos perciben el espacio público como adecuado, pero la observación del uso cotidiano indica lo contrario. Es posible que esto se deba a que, comparativamente, el área mencionada tiene más y mayores áreas verdes que otros distritos de Lima. Muchos de los nuevos residentes provienen de lugares en donde la situación es precaria, por lo que se percibe el estado actual como uno de mejora.

El establecimiento de una tradición implica la permanencia en el tiempo de una cierta actividad trascendente. La existencia de tradiciones contribuye a la cohesión social y al establecimiento de una identidad grupal (Huffschmid, 2012), en esto radica su importancia (Zaiatz-Crestani y Martins de Araújo-Klein, 2016). Dada la reciente movilidad de vecinos en el área estudiada, las pocas costumbres existentes aún distan de convertirse en tradiciones. El barrio aún se encuentra en un proceso de densificación y, adicionalmente, muchos de los nuevos departamentos son comprados para ser dados en alquiler, por lo que la percepción de los propios vecinos es la de una población que no necesariamente permanecerá en la zona. Ante esta situación, poco puede hacer la arquitectura pública. Mientras se de este proceso de cambio intenso en el vecindario, el establecimiento de tradiciones no se podrá dar, puesto que se requiere una cierta estabilidad que permita la repetitividad (Zaiatz-Crestani y Martins de Araújo-Klein, 2016). Cuando el proceso de cambio del vecindario finalice, y si las familias permanecen en los departamentos por períodos largos de tiempo, podrá producirse una identidad barrial. Sin embargo, existe un segundo factor que previene el establecimiento de tradiciones, la ausencia de una infraestructura pública adecuada que permita a los vecinos desarrollar actividades individuales o grupales al exterior. El actual uso de las bermas, por ejemplo, podría propiciar encuentros e intercambio entre los vecinos, si se tratara de un espacio con lugares para permanecer y desplazarse con comodidad; el hecho que sea un espacio improvisado y de difícil acceso peatonal, obliga a las personas a limitar su uso. En este caso, el diseño urbano y arquitectónico de los espacios ya existentes puede colaborar (Beer et al., 2003), teniendo en cuenta las dinámicas preestablecidas, a mejorar las condiciones para que estas se den de una mejor manera.

El ejemplo de la Residencial San Felipe muestra cómo -luego de un tiempo en el que los vecinos logren conocerse, y con espacios públicos adecuados- algunas de las costumbres, como festividades religiosas o eventos distritales, pueden volverse tradiciones.

El adecuado diseño de la infraestructura también tiene una injerencia en el establecimiento de hábitos que redunden en mejoras para el vecindario. Que los peatones utilicen los cruceros peatonales, que la basura esté en los lugares que corresponde y que la presencia de visitantes externos a la zona no interrumpa las actividades cotidianas de los vecinos, puede ser controlado con el diseño adecuado de cruceros, el emplazamiento de basureros, bancas, farolas y mobiliario urbano adecuado; y, en general, por el entendimiento por parte de diseñadores y promotores del espacio público como un complemento de la vivienda.

El espacio público juega un papel importante en el establecimiento de una memoria barrial colectiva. La comparación con los vecinos de la Residencial San Felipe habla de una memoria del lugar ligada al espacio, que permite establecer grupos e identidades; cuando el espacio no permite la permanencia, esto es casi imposible. La necesidad de darle un mejor tratamiento a los espacios públicos aumenta cuando se considera que, ante la densificación de los lotes y la dramática disminución del área de la vivienda en relación con las casas unifamiliares que estas reemplazan, ya no se puede confiar en que el interior de la vivienda 
sea suficiente para garantizar el cumplimiento satisfactorio de todas las actividades de los individuos. $\mathrm{Si}$ antes los niños jugaban en los patios o jardines traseros de sus casas, en la actualidad deben buscar otros espacios para hacerlo. El espacio público debe cumplir la doble tarea de cubrir las carencias físicas de un espacio que ya no existe en la vivienda, y de funcionar como lugar de encuentro que propicie las actividades sociales.

Ante el crecimiento urbano acelerado y la densificación, común a muchas ciudades en expansión y consolidación, queda preguntar ¿cómo actuar ante la situación dada y otorgar al espacio público un mayor protagonismo?

\section{Referencias}

Agudelo-Castañeda, J. (2011). Intangible. Acontecimiento urbano: su estructura simbólica y valor cultural. Bogotá: Oficina de Publicaciones, Universidad de La Salle.

Arendt, H. (2011/1958). Vita activa. Milano: Bompiani.

Bailly, A. S. (1979). La percepción del espacio urbano. Madrid: Instituto de Estudios de Administración Local.

Beer, A. B., Delshammar, T., y Schildwatch, P. (2003). A Changing Understanding of the Role of Greenspace in High-Density Housing: A European Perspective. Built Environment, 132-143.

Borja, J., y Muxí, Z. (2000). El espacio público, ciudad y ciudadania. Barcelona: Electa.

Brillenbourg, A., Feireiss, K., y Klumpner, H. (2005). Informal city. Caracas Case. München: Prestel.

Carrión, M. F. (2007). Espacio público: punto de partida para la alteridad. Quito: Flacso.
Cuervo-Calle, J. J., y Herrán-Cuartas, C. (2013). La casa en el parque: expresiones domésticas en el espacio público. Cuadernos de Vivienda y Urbanismo, 6(12), 228-247.

Cullen, G. (1974). El paisaje urbano. Tratado de estética urbana. Barcelona: Editorial Blume.

Franck, K. A., y Stevens, Q. (2007). Loose Space. Possibility and Diversity in Urban Life. Nueva York: Routledge.

Gehl, J. (2006/2004). La humanización del espacio urbano. La vida social entre los edificios. Barcelona: Reverté.

Groat, L., y Wang, D. (2002). Architectural Research Methods. Nueva York: John Wiley \& Sons.

Huffschmid, A. (2012). From the City to lo Urbano: Exploring Cultural Production of Public Space in Latin America. Iberoamericana, XII(45), 119-136.

Jacobs, J. (2011/1961). The Death and Life of Great American Cities. Nueva York: Modern Library.

Johnson, S. (2001). Sistemas Emergentes. O qué tienen en común hormigas, neuronas, ciudades y software. Madrid: Fondo de Cultura Económica.

Krier, R. (1981). El espacio urbano. Barcelona: Gustavo Gili S.A.

Lefebvre, H. (1973/1970). La rivoluzione urbana. Roma: Armando Armando.

Lima cómo vamos. (2013). Encuesta Lima cómo vamos 2012. Recuperado de http://www.limacomovamos.org/cm/wp-content/uploads/2014/09/ EncuestaLCV.pdf

Lima cómo vamos. (2018). VIII Informe de percepción sobre calidad de vida en Lima y Callao. Lima: Asociación Unacem. 
López, F. (2011). Boom limeño en la construcción. Business negocios en el Perú, (189), 30-32.

Ludeña-Urquizo, W. (2004). Lima. Historia y urbanismo en cifras. 1821-1970. Lima: Universidad Nacional de Ingeniería.

Lynch, K. (2001/1984). La imagen de la ciudad. Barcelona: Gustavo Gili.

Páramo, P., Burbano, A., Jiménez-Domínguez, B., Barrios, V., Pasquali, C., Vivas, F., ... Moyano, E. (2018). La habitabilidad del espacio público en las ciudades de América Latina. Avances en psicología Latinoamericana, 36(2), 345-362.

Páramo, P., y Burbano-Arroyo, A. (2014). Los usos y la apropiación del espacio público para el fortalecimientode la democracia. Revista de Arquitectura, 16, 6-15.

Pérez-Bourzac, M. T. (2018). El espacio público contemporáneo en la ciudad del siglo XXI. ¿Crisis o transformación? ACE Architecture, City and Environment, 12(36), 131-140.

Roca-Mora, A. (2013). El boom inmobiliario. Poder, (18), 66-76.
Rowe, C., y Koetter, F. (1998/1981). Ciudad collage. Barcelona: Gustavo Gili.

Soja, E. W. (2000). Postmetrópolis. Londres: Wiley.

Tokeshi, J., y Takano, G. (2007). Espacio público en la ciudad popular: reflexiones y experiencias desde el sur. Estudios Urbanos. Recuperado de http://209.177.156.169/libreria_cm/archivos/ pdf_869.pdf

Trachana, A. (2008). La evolución de la forma del espacio público. Buenos Aires: Nobuko.

Urban-Think Tank. (2013). Torre David. Informal Vertical Communities. Zürich: Lars Müller Publishers.

Vega-Centeno, P. (2017). La desigualdad invisible: el uso cotidiano de los espacios públicos en la Lima del siglo XXI. Territorios, (36), 23-46.

Zaiatz-Crestani, A. M., y Martins de Araújo-Klein, R. M. (2016). Espaço, imagem e memória: adensamento dos conteúdos coletivos à materialidade da cidade. Territorios, (36), 139-157. 